\title{
Analysis of Injection-Locked Antenna Array Including Mutual Coupling Effects
}

\author{
Kun-Chou Lee and Tah-Hsiung Chu, Member, IEEE
}

\begin{abstract}
In this paper, the injection locking performance analysis of dipole antenna array with each element loaded with a two-terminal oscillator is presented. The analysis is based on the nonlinear model of oscillator and the linear model of antenna array considering mutual coupling effects. The locking range of injection signal and the array radiated power are obtained by solving an equivalent multiport network. In general, the solutions include stable and unstable solutions. The Routh-Hurwitz stability criterion is then applied to remove the unstable solutions. Numerical results show that the array performance such as frequency locking range and radiated power by taking into account the array mutual coupling effects is quite different from that of an isolated antenna element. In addition, the influence of antenna element spacing upon array locking parameters in this paper is found to be consistent with other existing theories.
\end{abstract}

Index Terms-Injection locked oscillators (ILO), loaded antennas, mutual coupling.

\section{INTRODUCTION}

$\mathbf{N}$ ONLINEAR lumped loads are often attached to the input terminals of antennas to yield the desired scattering characteristics. The lumped loads may include passive and active microwave semiconductor devices. There have been many studies for the analyses of loaded antenna array with passive nonlinear lumped loads including mutual coupling effects [1]-[4]. In some applications, the nonlinear loads may involve active devices. For example, one can construct a millimeter spatial combiner using active array structure with each element consisting of an antenna and a solid-state oscillator. In order to coherently combine these solid-state sources to generate large output power, the oscillators are usually injection-locked to a reference signal.

As each element of the antenna array is loaded with an active device, the system becomes autonomous and the analysis is quite different from that of passive loads. Although many types of active arrays are experimentally studied, most of their theoretical analyses are based on linear models [5]-[18]. In [19] and [20], a nonlinear analysis approach is proposed to analyze the characteristics of an injection-locked oscillator (ILO) and injection-locked antenna (ILA) including locking range and output power. For an injection-locked antenna array (ILAA), each element of the array may receive the mutual coupling signals from

Manuscript received May 20, 2002; revised December 14, 2003. This work was supported in part by the National Science Council of Taiwan, R.O.C., under Grants 91-2219-E-002-030, 93-2752-E-002-001-PAE, and in part by the Ministry of Education of Taiwan, R.O.C., under Grant 89-E-FA06-2-4-6.

K.-C. Lee is with the Department of Systems and Naval Mechatronic Engineering, National Cheng-Kung University, Tainan, Taiwan, R.O.C. (e-mail: kclee@mail.ncku.edu.tw).

T.-H. Chu is with the Department of Electrical Engineering, National Taiwan University, Taipei, Taiwan, R.O.C. (e-mail: thc @ew.ee.ntu.edu.tw).

Digital Object Identifier 10.1109/TAP.2004.835236 other antenna elements. These coupling signals then have effects on the array performance including locking range and radiated power.

In this paper, the scattering and radiating analysis of dipole antenna array with each element loaded with a two-terminal oscillator is presented. The active antenna array is illuminated by an external injection plane wave. Since all the equivalent exciting sources at each antenna terminal are in coherence, the ILAA is synchronized to injection signal frequency. The analysis is then equivalent to solving a multiport network circuit with each port in shunt with a two-terminal oscillator and an equivalent exciting source. The multiport network parameters of an antenna element are obtained by the moment method. Since it is difficult to achieve an analytic time-domain model of oscillator, another approach different from that given for analyzing passive nonlinear load case is proposed to solve the resulting equivalent multiport network circuit.

In the analysis for each oscillator element, a nonlinear circuit equation is formulated in the frequency domain to describe the two-terminal ILO. Its output power can be solved accordingly. However, due to the property of ILO nonlinear equation, the resulting solutions include stable and unstable solutions in general. A characteristic equation with the power of $2 N$ is derived for an $N$-element ILAA including array mutual coupling effects. The Routh-Hurwitz stability criterion [21] is then applied to verify the stability property for every possible solution. Numerical results show that the frequency locking range by taking into account the array mutual coupling effects is quite different from that of an isolated antenna element. In addition, the influence of antenna element spacing upon array locking parameters is also discussed and compared with other existing theories [22]-[32].

In the following, the formulation of an ILAA including mutual coupling effects is described in Section II. Numerical simulation results are shown in Section III. Discussion and comparison with other existing theories are given in Section IV. Finally, the conclusion is given in Section V. In addition, the Routh-Hurwitz stability criterion is given in the Appendix.

\section{FORMULATION}

An ILAA is shown in Fig. 1(a) that consists of $N$ elements of nonlinearly loaded dipole antennas. Each isolated antenna element has an equivalent circuit as in Fig. 1(b). In Fig. 1(b), $Y(\omega)$ is the complex input admittance of the dipole antenna, $I_{e q}$ is the amplitude of the antenna short-circuit current induced by the incident field $E_{i}$, and $Y_{D}(V, \omega)$ is the admittance of a two-terminal active diode. 


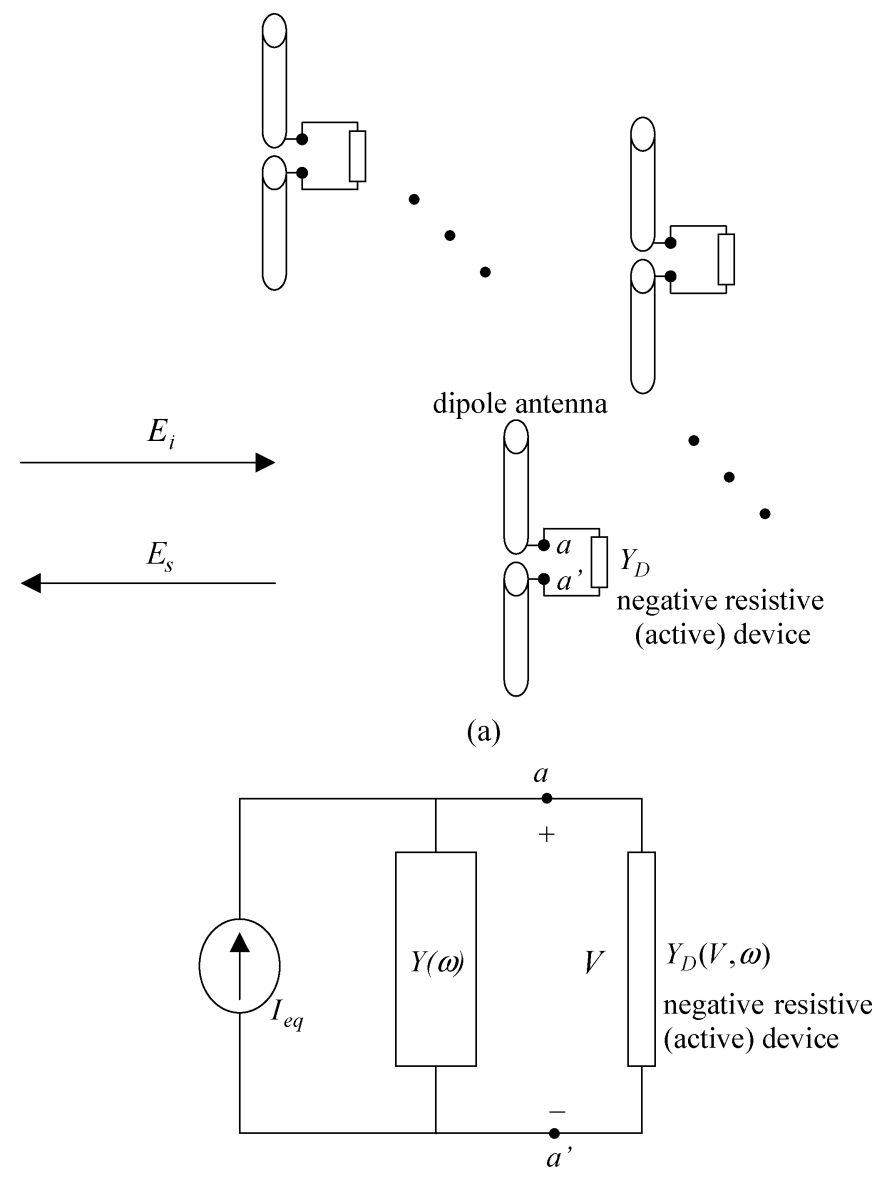

(b)

Fig. 1. Schematic diagram of (a) an injection-locked dipole antenna array and (b) the equivalent circuit of each isolated antenna element.

Therefore, the equivalent circuit of an ILAA can be represented as shown in Fig. 2, where $\overline{\bar{Y}}(\omega)$ denotes the antenna array input admittance matrix including the mutual coupling effects and $I_{e q_{i}} e^{j \alpha_{i}}$ is the short-circuit current phasor of the $i$ th antenna element induced by the incident field $E_{i}$ with frequency $\omega_{i n j}$.

As the incident field is absent, it is a free-running antenna array with radiated electromagnetic energy at resonant frequency $\omega_{0}$. The total array admittance is hence zero, i.e.

$\overline{\bar{Y}}_{T}(V, \omega)=\left[\begin{array}{cccc}Y_{11}+Y_{D_{1}} & Y_{12} & \cdots & Y_{1 N} \\ Y_{21} & Y_{22}+Y_{D_{2}} & \cdots & Y_{2 N} \\ \vdots & \vdots & \cdots & \vdots \\ Y_{N 1} & Y_{N 2} & \cdots & Y_{N N}+Y_{D_{N}}\end{array}\right]=\overline{\overline{0}}$

where $Y_{D_{i}}, i=1,2, \ldots, N$, is the admittance of the active device at the $i$ th antenna terminal. $Y_{i, j}$ is the $(i, j)$ th element in $\overline{\bar{Y}}_{T}$ to account for the input admittance of the $i$ th antenna as $i=j$, and the mutual coupling effect between the $i$ th and $j$ th antennas as $i \neq j$. The admittance of a two-terminal active diode at the fundamental harmonic frequency can be characterized by [33], [34]

$$
Y_{D}(V, \omega)=\left(G_{n 0}+G_{n 2} V^{2}\right)+j \omega\left(C_{n 0}+C_{n 2} V^{2}\right)
$$

where $V$ is the amplitude of the output response. For practical semiconductor devices, $G_{n 0}$ is negative and $G_{n 2}$ is positive.
As the array injection-locked state is achieved, all the dynamic frequencies are synchronized with $\omega_{\text {inj }}$, since all the equivalent exciting current sources at the antenna input terminals are coherent. The circuit equation of ILAA becomes

$$
\overline{\bar{Y}}_{T} \bar{V}=\bar{I}_{e q}
$$

or

$$
\begin{gathered}
{\left[\begin{array}{cccc}
Y_{11}+Y_{D_{1}} & Y_{12} & \cdots & Y_{1 N} \\
Y_{21} & Y_{22}+Y_{D_{2}} & \cdots & Y_{2 N} \\
\vdots & \vdots & \cdots & \vdots \\
Y_{N 1} & Y_{N 2} & \cdots & Y_{N N}+Y_{D_{N}}
\end{array}\right] \times} \\
\\
\end{gathered}
$$

The stability criteria for ILAA can then be derived in the following based on the same procedure given in [19], except that the scalar variables are replaced by vector representations. They are given as

$$
\begin{gathered}
\sum_{j=1}^{N}\left[\left(G_{T_{i j}}+\frac{\partial G_{T_{i j}}}{\partial V_{j}} V_{j}+s \frac{\partial B_{T_{i j}}}{\partial \omega}\right) \frac{\delta V_{j}}{V_{j}}\right. \\
\left.-\left(B_{T_{i j}}-s \frac{\partial G_{T_{i j}}}{\partial \omega}\right) \delta \phi_{j}\right]=0 \\
\sum_{j=1}^{N}\left[\left(B_{T_{i j}}+\frac{\partial B_{T_{i j}}}{\partial V_{j}} V_{j}-s \frac{\partial G_{T_{i j}}}{\partial \omega}\right) \frac{\delta V_{j}}{V_{j}}\right. \\
\left.+\left(G_{T_{i j}}+s \frac{\partial B_{T_{i j}}}{\partial \omega}\right) \delta \phi_{j}\right]=0
\end{gathered}
$$

where $i=1,2, \ldots, N$, s denotes $\partial / \partial t$, and $Y_{T_{i j}}=G_{T_{i j}}+$ $j B_{T_{i j}}$ is the $(i, j)$ th element of $\overline{\bar{Y}}_{T}$ in (3).

The existing nontrivial solutions require that the determinant of the coefficient matrix of the above $2 \mathrm{~N}$ equations to be zero, i.e.

$$
\operatorname{det}\left[\begin{array}{ccccc}
a_{11} & b_{11} & \cdots & a_{1 N} & b_{1 N} \\
c_{11} & d_{11} & \cdots & c_{1 N} & d_{1 N} \\
\vdots & \vdots & \ddots & \vdots & \vdots \\
a_{N 1} & b_{N 1} & \cdots & a_{N N} & b_{N N} \\
c_{N 1} & d_{N 1} & \cdots & c_{N N} & d_{N N}
\end{array}\right]=0
$$

where

$$
\begin{aligned}
a_{i j} & =G_{T_{i j}}+\frac{\partial G_{T_{i j}}}{\partial V_{j}} V_{j}+s \frac{\partial B_{T_{i j}}}{\partial \omega} \\
b_{i j} & =-B_{T_{i j}}+s \frac{\partial G_{T_{i j}}}{\partial \omega} \\
c_{i j} & =B_{T_{i j}}+\frac{\partial B_{T_{i j}}}{\partial V_{j}} V_{j}-s \frac{\partial G_{T_{i j}}}{\partial \omega} \\
d_{i j} & =G_{T_{i j}}+s \frac{\partial B_{T_{i j}}}{\partial \omega} .
\end{aligned}
$$

For stable solutions, all the roots of (7) must lie in the lefthalf $s$-plane. Since (7) is a polynomial equation of $s$ with the power of $2 N$, the Routh-Hurwitz stability criterion [21], which is given in the Appendix can be applied to determine the stable 


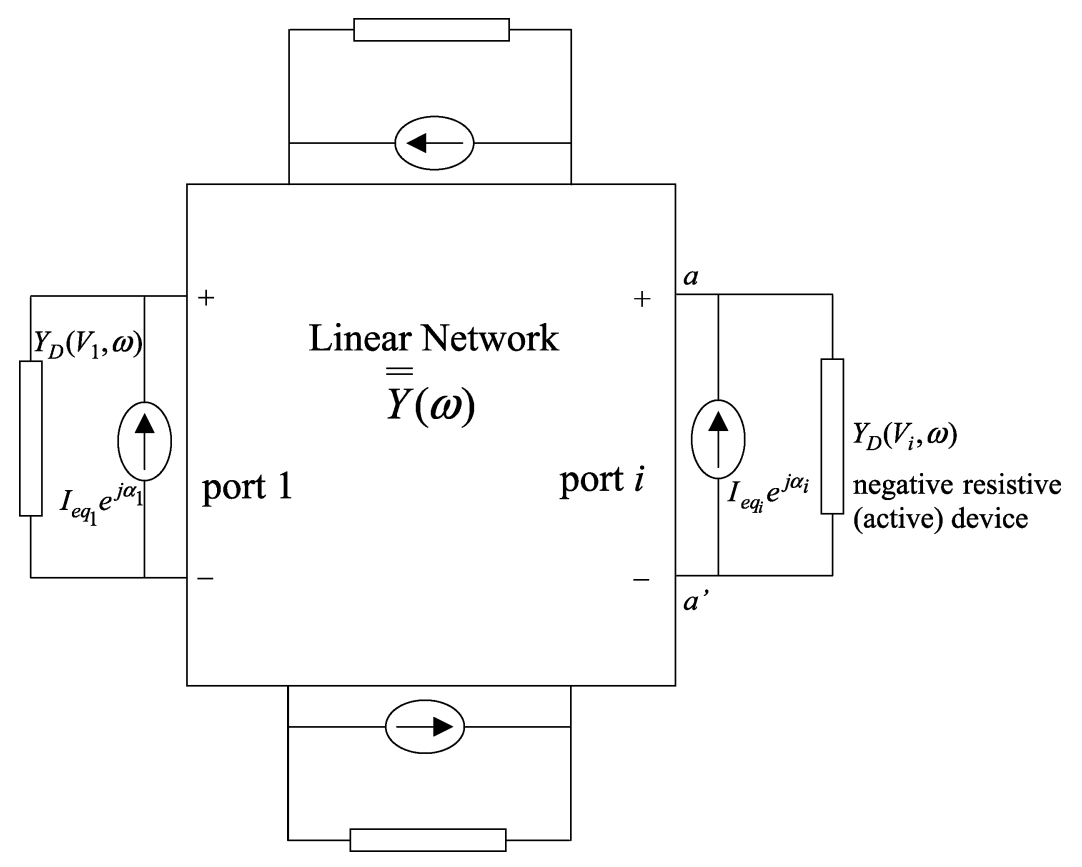

Fig. 2. The equivalent circuit of Fig. 1(a).

conditions for the solutions of (4). The array locking parameters such as frequency locking range and radiated power can then be determined uniquely from (4), (7), and (8) by using the Routh-Hurwitz stability criterion.

In the special case of single ILA, (5) and (6) become two nonlinear equations as

$$
\begin{aligned}
& \left(G_{T}+\frac{\partial G_{T}}{\partial V} V+s \frac{\partial B_{T}}{\partial \omega}\right) \frac{\delta V}{V}-\left(B_{T}-s \frac{\partial G_{T}}{\partial \omega}\right) \delta \phi=0 \\
& \left(B_{T}+\frac{\partial B_{T}}{\partial V} V-s \frac{\partial G_{T}}{\partial \omega}\right) \frac{\delta V}{V}+\left(G_{T}+s \frac{\partial B_{T}}{\partial \omega}\right) \delta \phi=0 .
\end{aligned}
$$

In order to have variables $\delta V / V$ and $\delta \phi$ with nontrivial solution, the determinant of coefficients in (9) and (10) is required to be zero, or

$$
\begin{gathered}
\left|\frac{\partial Y_{T}}{\partial \omega}\right|^{2} s^{2}+\left[2\left(G_{T} \frac{\partial B_{T}}{\partial \omega}-B_{T} \frac{\partial G_{T}}{\partial \omega}\right)\right. \\
\left.+\left(\frac{\partial G_{T}}{\partial V} \frac{\partial B_{T}}{\partial \omega}-\frac{\partial B_{T}}{\partial V} \frac{\partial G_{T}}{\partial \omega}\right) V\right] s \\
+\left[G_{T}\left(G_{T}+\frac{\partial G_{T}}{\partial V} V\right)+B_{T}\left(B_{T}+\frac{\partial B_{T}}{\partial V}\right)\right]=0 .
\end{gathered}
$$

The stable condition then requires all roots of $s$ lying in the left-half complex plane, i.e.

$$
\begin{aligned}
2\left(G_{T} \frac{\partial B_{T}}{\partial \omega}-B_{T} \frac{\partial G_{T}}{\partial \omega}\right) & \\
& +\left(\frac{\partial G_{T}}{\partial V} \frac{\partial B_{T}}{\partial \omega}-\frac{\partial B_{T}}{\partial V} \frac{\partial G_{T}}{\partial \omega}\right) V>0 \\
G_{T}\left(G_{T}\right. & \left.+\frac{\partial G_{T}}{\partial \omega} V\right)+B_{T}\left(B_{T}+\frac{\partial B_{T}}{\partial V}\right)>0 .
\end{aligned}
$$

One can determine its locking performance by evaluating the all possible solutions of (3) based on (12) and (13).

\section{NUMERICAL EXAMPLES}

In this section, numerical examples are given to illustrate the analyses described in Section II for single ILA and ILAA, respectively. For simplicity, the dipole antennas are considered, although there is no limitation on the types of antenna. In the simulation, the dipole antenna is of length $10 \mathrm{~cm}$ and diameter $0.135 \mathrm{~cm}$. It is loaded with a two-terminal oscillator with the characteristics given by (2). For the simplicity of consideration, the capacitance term in (2) is ignored and parameters $G_{n 0}$ and $G_{n 2}$ are $-0.04 \mathrm{~A} / \mathrm{V}$ and $0.24 \mathrm{~A} / \mathrm{V}^{3}$, respectively.

In the free-running case, i.e., the incident field $E_{i}=0$, the active device oscillates at the antenna resonant frequency $\omega_{0}=$ $1.405 \mathrm{GHz}$, and the free-running voltage $V_{0}$ at the antenna input terminal is $0.33 \mathrm{~V}$ by solving (1).

As the dipole antenna is illuminated by an incident wave $E_{i}=1.0 \mathrm{~V} / \mathrm{m}$, the active antenna is then synchronized with the injection signal frequency $\omega_{i n j}$. The resulting voltage $V$ at the antenna input terminal can be determined by solving (3), and the stable solutions should satisfy the two inequalities of (12) and (13). Fig. 3 shows the normalized voltage at the antenna input terminal at different injection signal frequencies. There are three possible solutions of $V^{2}$ of (3) shown by three solid lines. However, there is only one stable solution of $V^{2}$ satisfying the stable criteria given in (12) and (13) indicated by the unstable region bounded by a dashed line. The locking range of ILA corresponding to the frequency range determined by the top oval-shaped solid line and the dashed line is within $0.978 \omega_{0}$ and $1.025 \omega_{0}$.

In the simulation of ILAA, two parallel identical ILAs given above are considered for the simplicity of analysis. The incident field is also $E_{i}=1.0 \mathrm{~V} / \mathrm{m}$. The resulting voltages $V_{1}$ and $V_{2}$ at each antenna input terminal can then be determined by solving (3), while the stable condition can be verified by (7), (8), and the Routh-Hurwitz stability criterion. 


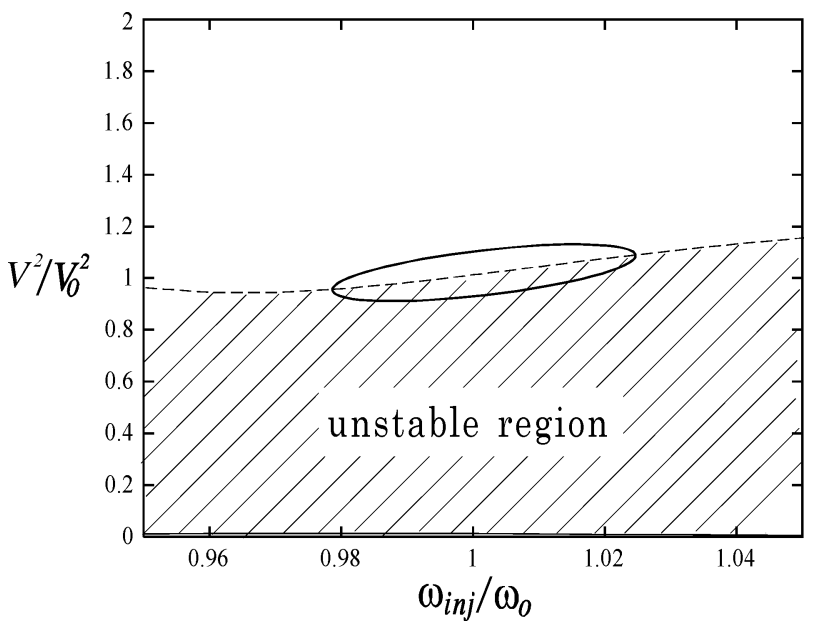

Fig. 3. Simulation results of the antenna input terminal voltage of an ILA at different injection frequencies. The vertical and horizontal axes are normalized to the free-running results. The dashed line is given by inequalities (12) and (13).

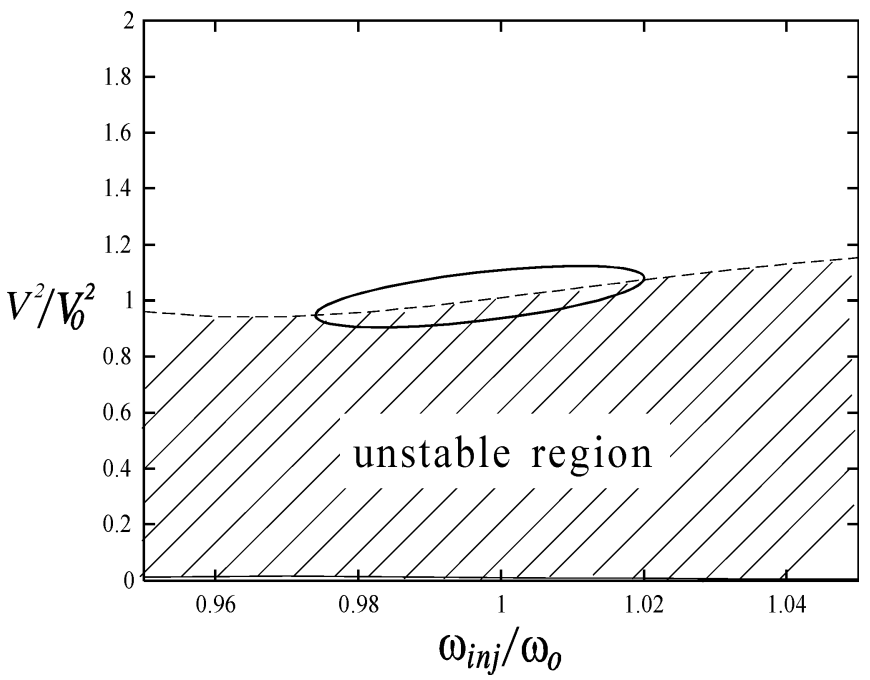

Fig. 4. Simulation results of the antenna input terminal voltage of a two-dipole ILAA with dipole spacing $42.7 \mathrm{~cm}$.

Fig. 4 shows the normalized voltage at each antenna input terminal of ILAA at different injection signal frequencies with dipole element spacing $42.7 \mathrm{~cm}$. Note $V_{1}=V_{2}=V$, due to the symmetry of the two-dipole arrangement. For convenience, all the resulting voltages and injection signal frequencies are normalized to those of the free-running case given in Fig. 3. The locking range in Fig. 4 shows between $0.975 \omega_{0}$ and $1.02 \omega_{0}$. This slight change of locking range is due to the mutual coupling effects between two dipoles. In fact, each element of the antenna array receives the mutual coupling signals from other antenna elements. These coupling signals then have influence on the ILAA locking performance such as locking range and radiated power.

As the dipole spacing is reduced to $10 \mathrm{~cm}$, the simulation results are shown in Fig. 5. It shows that the locking range is

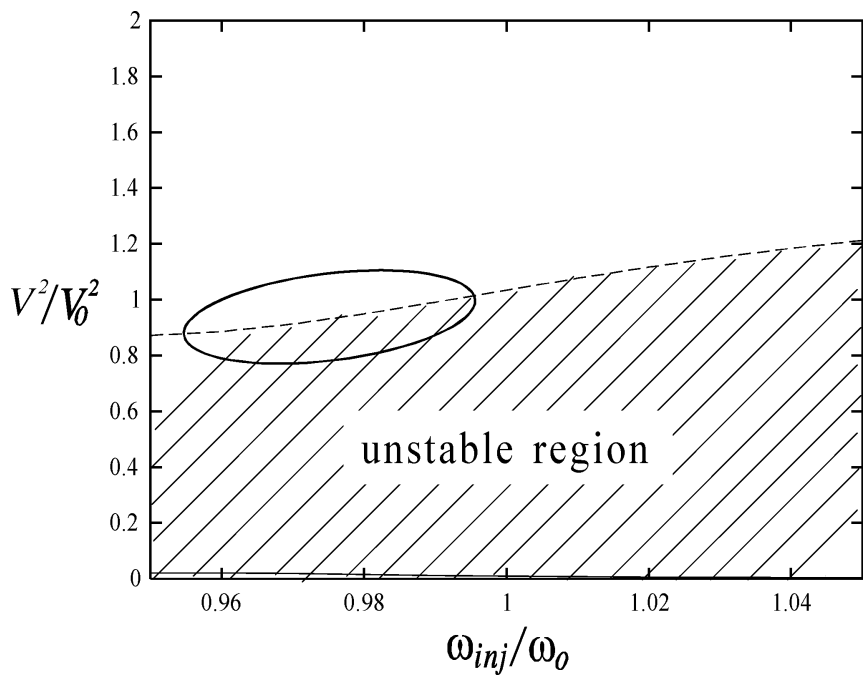

Fig. 5. Simulation results of the antenna input terminal voltage of a two-dipole ILAA with dipole spacing $10 \mathrm{~cm}$.

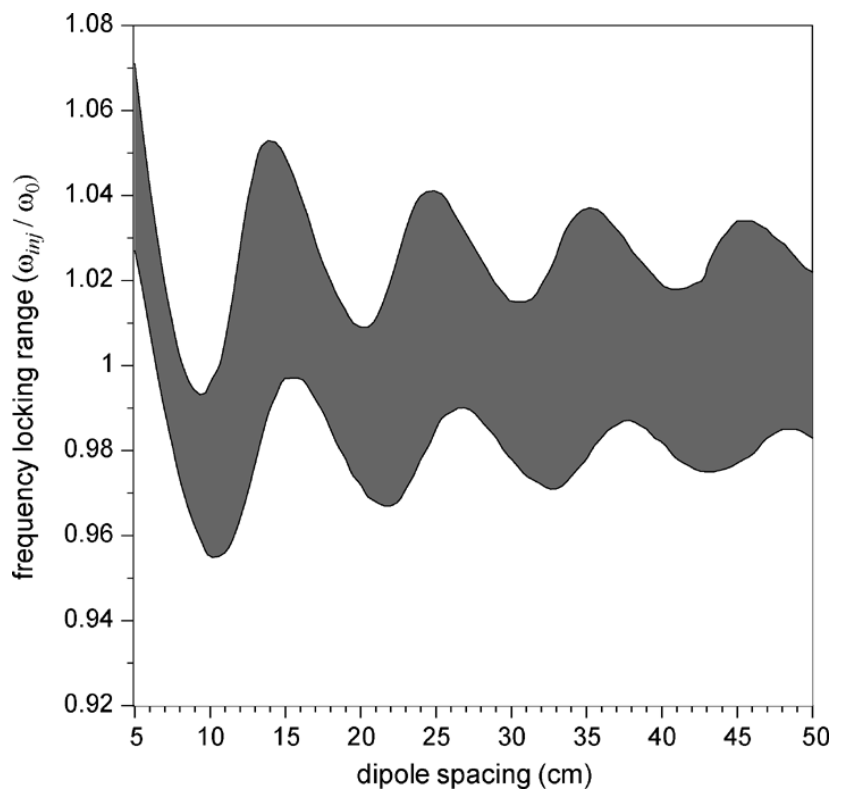

Fig. 6. The frequency locking range (as shown the shadow region) of a two-dipole ILAA described above for different dipole spacing.

changed to be within $0.955 \omega_{0}$ and $0.996 \omega_{0}$, which is quite different from that of Fig. 4. This is because the mutual coupling effect in this case is quite different from that of Fig. 4.

Fig. 6 shows the frequency locking range (as the shadow region) of the two-dipole ILAA described above under different dipole spacing. It also shows that different array geometries have different mutual coupling effects and thus lead to different frequency locking ranges. The variations of frequency locking range in Figs. 4-6 will be discussed in detail in the following section.

The above numerical examples are simulated using Fortran-90 codes on a PC with Intel Pentium $2.4 \mathrm{GHz}$ CPU. The computation time for each point of injection locking frequency in Fig. 3 is about $0.7 \mathrm{~s}$. The computation time for each point of injection locking frequency in Fig. 4 or 5 is about $1.0 \mathrm{~s}$ due to the calculation of mutual coupling effects. 


\section{DISCUSSION}

There have been many studies involving the stability of mutual injection locked oscillators with and without external injection [22]-[32]. In this section, the formulations and numerical results described above are interpreted based on the results of [23] and [31].

In [23], a linear array of $N$ oscillator antennas is considered with the assumption of nearest neighboring mutual coupling. These $N$ oscillator antennas are mutually injection locked without external injection signal. The interaction between the $i$ th and the $j$ th oscillator antennas is characterized by a dimensionless complex coupling coefficient $\kappa_{i j}$ with the magnitude and phase given by [23]

$$
\kappa_{i j}=\left\{\begin{array}{cl}
\varepsilon e^{-j \Phi}, & |i-j|=1 \\
0, & \text { otherwise }
\end{array}\right.
$$

where $\varepsilon$ and $\Phi$ are the magnitude and phase terms of the coupling coefficient $\kappa_{i j}$. According to [23], for each oscillator antenna, there exists a frequency shift with respect to its free-running resonant frequency, and this frequency shift will vary and oscillate as the sine of the coupling phase $\Phi$. It should be noted that $\Phi$ depends on the array geometry, i.e., array mutual coupling effects. On the other hand, according to [31], the effective locking range of a mutual ILAA without external injection signal will vary as the cosine of the coupling phase $\Phi$ and then become zero as $\Phi$ is an odd multiple of $90^{\circ}$.

It should be emphasized that the results in [23] and [31] are under no external injection signals. However, both the mutual and external injection signals exist for each oscillator antenna in this paper. In the two-dipole ILAA described in Section III, the total signals injected into a given oscillator antenna can be divided into two parts, i.e., a large component from the external injection plane wave and a small component of mutual coupling from the neighboring oscillator antenna. The large component is due to the direct incidence from external injection and is constant. On the other hand, the small component is due to mutual coupling from the other dipole element and will differ in phase depending on the coupling phase, i.e., the dipole spacing. One can now add these two components together. It will become the vector sum of a large fixed vector and a small vector rotating as a function of the coupling phase. The phase of the resulting vector will oscillate with the coupling phase, i.e., the dipole spacing, and then produce oscillating ensemble frequency as those given in [23]. These phenomena can be observed from Fig. 6. It should be noted that the phase of the resulting vector can never reach $90^{\circ}$ since the external injection signal strength is in general larger than the mutual injection signal strength. This is the reason why the locking bandwidth in Fig. 6 never becomes zero as those given in [31].

\section{CONCLUSION}

In this paper, the locking performance of ILAA is analyzed with the consideration of array mutual coupling effects. The analysis is formulated in the frequency domain because the nonlinear characteristics of oscillator is given in the frequency domain. A characteristic equation with the power of $2 N$ is derived to describe the stable condition of an $N$-element ILAA. The
Routh-Hurwitz stability criterion is then applied to verify the stability property of each possible solution.

Numerical simulation results show that the array performance by taking into account the array mutual coupling effects is quite different from that of an isolated antenna element. The array locking range is shifted as there exists strong array mutual coupling effect; however, the array locking bandwidth is almost unchanged. These results are consistent with other existing studies [23], [31]. The analysis given in this paper is useful in the application of ILAA in the areas of communication system and remote sensing.

\section{APPENDIX \\ ROUTH-HURWITZ STABILITY CRITERION}

The Routh-Hurwitz stability criterion [21] is described in detail. It is a method for determining how many zeros of a polynomial are in the right-half plane or on the imaginary axis.

Consider a polynomial equation to represent a system characteristic equation as

$$
f(s)=a_{n} s^{n}+a_{n-1} s^{n-1}+\cdots+a_{1} s+a_{0}=0 .
$$

A Routh array is then constructed with the first two rows produced by arranging the coefficients $a_{n}, a_{n-1}, \ldots, a_{0}$ in order as

$$
\begin{array}{ccccccc}
s^{n} & : & a_{n} & a_{n-2} & a_{n-4} & \cdots & 0 \\
s^{n-1} & : & a_{n-1} & a_{n-3} & a_{n-5} & \cdots & 0 \\
s^{n-2} & : & b_{1} & b_{2} & b_{3} & \cdots & 0 \\
s^{n-3} & : & c_{1} & c_{2} & c_{3} & \cdots & 0 \\
\vdots & : & & & & & \\
s^{1} & : & g_{1} & 0 & & & \\
s^{0} & : & h_{1} & 0 . & & &
\end{array}
$$

Each of the remaining entries $b_{i}, c_{i}, \ldots$ is found from the two rows above them according to the following relations:

$$
\begin{aligned}
& b_{1}=\frac{-1}{a_{n-1}}\left|\begin{array}{cc}
a_{n} & a_{n-2} \\
a_{n-1} & a_{n-3}
\end{array}\right|=\frac{1}{a_{n-1}}\left(a_{n-1} a_{n-2}-a_{n} a_{n-3}\right) \\
& b_{2}=\frac{-1}{a_{n-1}}\left|\begin{array}{cc}
a_{n} & a_{n-4} \\
a_{n-1} & a_{n-5}
\end{array}\right|=\frac{1}{a_{n-1}}\left(a_{n-1} a_{n-4}-a_{n} a_{n-5}\right) \\
& b_{3}=\frac{-1}{a_{n-1}}\left|\begin{array}{cc}
a_{n} & a_{n-6} \\
a_{n-1} & a_{n-7}
\end{array}\right|=\frac{1}{a_{n-1}}\left(a_{n-1} a_{n-6}-a_{n} a_{n-7}\right) \\
& c_{1}=\frac{-1}{b_{1}}\left|\begin{array}{cc}
a_{n-1} & a_{n-3} \\
b_{1} & b_{2}
\end{array}\right|=\frac{1}{b_{1}}\left(b_{1} a_{n-3}-b_{2} a_{n-1}\right) \\
& c_{2}=\frac{-1}{b_{1}}\left|\begin{array}{cc}
a_{n-1} & a_{n-5} \\
b_{1} & b_{3}
\end{array}\right|=\frac{1}{b_{1}}\left(b_{1} a_{n-5}-b_{3} a_{n-1}\right) .
\end{aligned}
$$

Calculations in each row are continued until only zero elements remain. In each of the last two rows the second and following elements are zero. It can be shown that the elements in any row can be multiplied by an arbitrary positive constant without affecting the results below. This is useful to simplify the arithmetic computation.

In summary, the Routh-Hurwitz stability criterion states the following.

1) A necessary and sufficient condition for stability is that there are no changes of sign in the elements of the first column of the array (A2).

2) The number of these sign changes is equal to the number of roots in the right-half $s$-plane. 
3) If the first element in a row is zero, it is replaced by a very small positive number $\varepsilon$, and the sign changes when $\varepsilon \rightarrow 0$ are counted after completing the array.

4) If all elements in a row are zero, the equation has roots in the right-half plane or on the imaginary axis, i.e., the system characterized by (A1) is unstable.

\section{ACKNOWLEDGMENT}

The authors would like to express their sincere gratitude to the reviewers for their helpful comments, especially the physical interpretations of the numerical simulation results in this paper.

\section{REFERENCES}

[1] K. C. Lee, "Analysis of large nonlinearly loaded antenna arrays under multi-tones excitation," Microwave Opt. Technol. Lett., pp. 319-323, June 2000.

[2] - "Analysis of nonlinearly loaded antenna array using the reflection algorithm," in IEEE AP-S Symp., July 2000.

[3] - "Two efficient algorithms for the analyzes of a nonlinearly loaded antenna and antenna array in the frequency domain," IEEE Trans. Electromagn. Compat., vol. 42, pp. 339-346, Nov. 2000.

[4] _ "Genetic algorithms based analyzes of nonlinearly loaded antenna arrays including mutual coupling effects," IEEE Trans. Antennas Propagat., vol. 51, pp. 776-781, Apr. 2003.

[5] L. Wanginger and V. Nalbandian, "Millimeter-wave power-combiner using quasioptical techniques," IEEE Trans. Microwave Theory Tech., vol. MTT-31, pp. 189-193, Feb. 1983.

[6] J. W. Mink, "Quasioptical power combining of solid-state millimeterwave sources," IEEE Trans. Microwave Theory Tech., vol. MTT-34, pp. 273-279, Feb. 1986.

[7] Z. B. Popovic, R. M. Weikle II, M. Kim, and D. B. Rutledge, "A 100MESFET planar grid oscillator," IEEE Trans. Microwave Theory Tech., vol. 39, pp. 193-200, Feb. 1991.

[8] E. R. Brown, C. D. Parker, K. M. Molvar, and K. D. Stephan, "A quasioptically stabilized resonant-tunneling-diode oscillator for the millimeterand submillimeter-wave regions," IEEE Trans. Microwave Theory Tech., vol. 40, pp. 846-850, May 1992.

[9] H. Kondo, M. Hieda, M. Nakayma, T. Tanaka, K. Osakabe, and K. Mizuno, "Millimeter and submillimeter wave quasioptical oscillator with multi-elements," IEEE Trans. Microwave Theory Tech., vol. 40, pp. 857-863, May 1992.

[10] R. M. Weikle II, M. Kim, J. B. Hacker, M. P. De Lisio, and D. B. Rutledge, "Planar MESFET grid oscillators using gate feedback," IEEE Trans. Microwave Theory Tech., vol. 40, pp. 1997-2003, Nov. 1992.

[11] A. Pance and M. J. Wengler, "Microwave modeling of 2-D active grid arrays," IEEE Trans. Microwave Theory Tech., vol. 41, pp. 20-28, Jan. 1993.

[12] K. D. Stephan, "Inter-injection-locked oscillators for power combining and phase arrays," IEEE Trans. Microwave Theory Tech., vol. MTT-34, pp. 1017-1025, Oct. 1986.

[13] D. Stephan and W. A. Morgan, "Analysis of inter-injection locked oscillators for integrated phase arrays," IEEE Trans. Antennas Propagat., vol. AP-35, pp. 771-781, July 1987.

[14] K. Chang, K. A. Hummer, and J. L. Klein, "Experiments on injection locking of active antenna elements for active phased arrays and spatial power combiners," IEEE Trans. Microwave Theory Tech., vol. 37, pp. 1078-1084, July 1989.

[15] R. A. York and R. C. Compton, "Quasioptical power combining using mutually synchronized oscillator arrays," IEEE Trans. Microwave Theory Tech., vol. MTT-39, pp. 1000-1009, June 1991.

[16] J. Birkeland and T. Itoh, "A 16-element quasioptical FET oscillator power combining array with external injection locking," IEEE Trans. Microwave Theory Tech., vol. 40, pp. 475-481, Mar. 1992.

[17] W. A. Shiroma, B. L. Shaw, and Z. B. Popovic, "A 100-transistor quadruple grid oscillator," IEEE Microwave Guided Wave Lett., vol. 4, pp. 350-351, Oct. 1994.

[18] Y. Shen, C. Laperle, N. Sangary, and J. Litva, "A new active array module for spatial power combiners and active antennas," IEEE Trans. Microwave Theory Tech., vol. 43, pp. 683-684, Mar. 1992.

[19] C. C. Huang and T. H. Chu, "Analysis of MESFET injection-locked oscillators in fundamental mode of operation," IEEE Trans. Microwave Theory Tech., vol. 42, pp. 1851-1857, Oct. 1994.

[20] _ "Radiating and scattering analyzes of a slot-coupled patch antenna loaded with a MESFET oscillator," IEEE Trans. Antennas Propagat., vol. AP-43, pp. 291-298, Mar. 1995.
[21] J. V. De Vegte, Feedback Control Systems. Englewood Cliffs, NJ: Prentice-Hall, 1986.

[22] R. A. York and R. C. Compton, "Measurement and modeling of radiative coupling in oscillator arrays," IEEE Trans. Microwave Theory Tech., vol. 41, pp. 438-444, Mar. 1993.

[23] R. A. York, "Nonlinear analysis of phase relationships in quasioptical oscillator arrays," IEEE Trans. Microwave Theory Tech., vol. 41, pp. 1799-1809, Oct. 1993.

[24] R. A. York and P. Liao, "A new phase-shifterless beam-scanning technique using arrays of coupled oscillators," IEEE Trans. Microwave Theory Tech., vol. 41, pp. 1810-1815, Oct. 1993.

[25] S. Nogi, J. Lin, and T. Itoh, "Mode analysis and stabilization of a spatial power combining array with strongly coupled oscillators," IEEE Trans. Microwave Theory Tech., vol. 41, pp. 1827-1837, Oct. 1993.

[26] R. A. York, P. Liao, and J. J. Lynch, "Oscillator array dynamics with broadband $N$-port coupling networks," IEEE Trans. Microwave Theory Tech., vol. 42, pp. 2040-2045, Nov. 1994.

[27] H. C. Chang, E. S. Shapiro, and R. A. York, "Influence of the oscillator equivalent circuit on the stable modes of parallel-coupled oscillators," IEEE Trans. Microwave Theory Tech., vol. 45, pp. 1232-1239, Aug. 1997.

[28] R. A. York and T. Itoh, "Injection- and phase-locking techniques for beam control," IEEE Trans. Microwave Theory Tech., vol. 46, pp. 1920-1929, Nov. 1998.

[29] R. J. Pogorzelski, P. F. Maccarini, and R. A. York, "A continuum model of the dynamics of coupled-oscillator arrays for phase shifterless beamscanning," IEEE Trans. Microwave Theory Tech., vol. 47, pp. 463-470, Apr. 1999.

[30] - "Continuum model of the dynamics of externally injection locked coupled oscillator arrays," IEEE Trans. Microwave Theory Tech., vol. 47, pp. 471-478, Apr. 1999.

[31] R. J. Pogorzelski, R. P. Scaramastra, J. Huang, R. J. Beckon, S. M. Petree, and C. M. Chavez, "A seven-element S-band coupled-oscillator controlled agile-beam phase array," IEEE Trans. Microwave Theory Tech., vol. 48, pp. 1375-1384, Aug. 2000.

[32] B. K. Meadows et al., "Nonlinear antenna technology," Proc. IEEE, vol. 90, pp. 882-897, May 2002.

[33] G. H. B. Hasson and K. I. Lundström, "Stability criteria for phase-locked oscillators," IEEE Trans. Microwave Theory Tech., vol. MTT-20, pp. 641-645, Oct. 1972.

[34] E. F. Calandra and A. M. Sommariva, "Stability analysis of injectionlocked oscillators in fundamental mode operation," IEEE Trans. Microwave Theory Tech., vol. MTT-29, pp. 1137-1144, Nov. 1981.

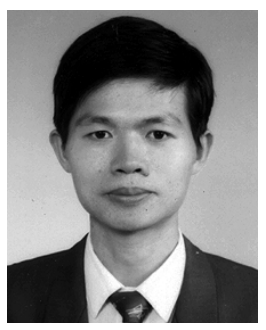

Kun-Chou Lee was born in Chia-yi, Taiwan, R.O.C., in 1966. He received the B.S., M.S., and Ph.D. degrees from National Taiwan University, Taipei, in 1989, 1991, and 1995, respectively, all in electrical engineering.

From 1995 to 1997, he was in military service. From 1997 to 1999, he was an Assistant Professor at Wu-Feng Institute of Technology. From 1999 to 2001, he was an Associate Professor at Shu-Te University. From 2002 to 2003, he was an Associate Professor at the National Kaohsiung University of Applied Sciences. In 2004, he joined the Faculty of the Department of Systems and Naval Mechatronic Engineering, National Cheng-Kung University, where he is now an Associate Professor. His research interests include microwave imaging, antennas, microwave circuits, and application of wireless technologies in land, oceanic, and underwater environments.

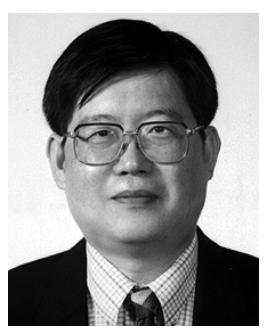

Tah-Hsiung Chu (M'87) received the B.S. degree from National Taiwan University, Taipei, Taiwan, R.O.C., in 1976 and the M.S. and Ph.D. degrees from the University of Pennsylvania, Philadelphia, in 1980 and 1983, respectively, all in electrical engineering.

From 1983 to 1986 , he was a Member of Technical Staff with the Microwave Technology Center, RCA David Sarnoff Research Center, Princeton, NJ. Since 1986, he has been on the Faculty of the Department of Electrical Engineering, National Taiwan University, where he is now a Professor of electrical engineering. His research interests include microwave-imaging systems and techniques, microwave circuits and subsystems, microwave measurements, and calibration techniques. 